\title{
Simulação de escoamentos viscosos utilizando um método SPH renormalizado
}

\section{Simulating viscous flow with a renormalized SPH method}

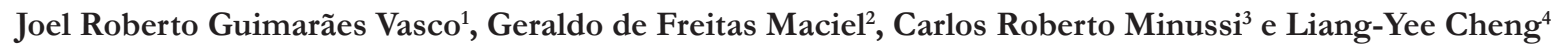

${ }^{1}$ Universidade Federal de Goiás (UFG) - Escola de Engenharia Civil (EEC)

$$
\text { joelvasco@ufg.br }
$$

${ }^{2}$ Universidade Estadual Paulista (Unesp), campus de Ilha Solteira/SP - Departamento de Engenharia Civil (DEC) maciel@dec.feis.unesp.br

${ }^{3}$ Universidxde Estadual Paulista (Unesp)), campus de Ilha Solteira/SP - Departamento de Engenharia Civil (DEC) minussi@dee.feis.unesp.br

${ }^{4}$ Universidade de São Paulo (USP) - Departamento de Engenharia de Construção Civil (PCC) cheng.yee@poli.usp.br

\section{RESUMO}

$O$ artigo investiga o comportamento numérico de escoamentos de fluidos newtonianos, em regime laminar e em superfície livre, usando um métodode partículas sem malha, lagrangiano, chamado SPH (Smoothed Particle Hydrodynamics). Aplicam-se correções ao método SPH original, como a renormalização do núcleo de suavização e do seu gradiente, a fim de minimiz̧ar o efeito da desorganização das partículas ao longo da simulação. Para validar o código numérico proposto, são empreendidos os estudo de casos: Poiseuille plano, Poisenille com superfície livre e o escoamento de Couette, comparando o perfil de velocidade numérico com soluções analíticas, tanto no regime estacionário quanto transiente. Finalmente, é analisado o problema tipo ruptura de barragens, comparando a evolução do alcance da frente numérica e experimental. Os resultados sugerem que o código SPH renormalizado proposto é capaz de reproduzir o escoamento de fluidos newtonianos em regime laminar.

Palavras Chave: Métodos sem malha. SPH. Renormalização. Escoamento laminar

\begin{abstract}
This paper evaluates the numerical behavior of laminar Newtonian fluid flows in a free surface, using a meshless method called SPH (Smoothed Particle Hydrodynamics). In order to minimize problems related to the position of the particles, some corrections are applied to the standard SPH method, such as kernel and gradient renormalizations. The code is validated by comparing the numerical velocity profile results with analytical ones, both in the steady and transient regime. The studied cases are a flat Poisenille, a free surface Poisenille, and a Couette flow. Finally, a dam break problem is analyzed, comparing the evolution and reach of the numerical and experimental wave. Based on the presented results, it can be concluded that the proposed SPH renormalized model is capable of reproducing laminar Newtonian fluid flows with free surface.
\end{abstract}

Keywords: Meshless methods. SPH. Renormalization. Laminar flow. 


\section{INTRODUÇÃO}

Escoamentos de fluidos newtonianos, em regime laminar, têm sido objeto de estudo há vários anos, pois necessitam da resolução da equação completa de Navier-Stokes. Para problemas com condições de contorno simples, existem soluções analíticas, enquanto que para casos mais complexos, no qual se enquadram a grande maioria dos problemas da Engenharia, normalmente recorrem-se aos modelos numéricos.

Em se tratando de modelos numéricos, ressalta-se sua crescente utilização como ferramentas de previsão do comportamento geral do escoamento, chegando, atualmente, a constituir elemento norteador de decisão de projeto. Neste contexto, os métodos sem malha (MSM) aparecem como opção com potencial de aplicação, tendo em vista suas vantagens em relação aos métodos numéricos tradicionais, que necessitam de uma malha para discretização do domínio. Uma dessas vantagens é a melhor capacidade de lidar com descontinuidades, que podem aparecer em problemas de superfície livre, por exemplo. Outra é que com a aproximação lagrangiana dos MSM, a utilização da derivada total dispensa o cálculo de termos convectivos, que são comumente encontrados em métodos Eulerianos e normalmente estão associados à difusão numérica. Adicionalmente, no caso particular do método sem malha SPH (Smoothed Particle Hydrodynamics), as condições de contorno cinemática e dinâmica na superfície livre não precisam ser impostas, sendo automaticamente satisfeitas (OGER et al., 2007), simplificando sobremaneira a complexidade do problema.

Há, no entanto, alguns inconvenientes para a aplicação do SPH a escoamentos de fluidos reais, notadamente em relação à discretização de termos laplacianos e a representação das condições de contorno na fronteira. Monaghan (2006), que simulou no seu código baseado no SPH o clássico escoamento de Couette, utiliza a própria expressão da viscosidade artificial para representar a viscosidade física do fluido, aplicando a técnica das fronteiras reativas às condições de contorno em paredes sólidas. Procedimento similar foi aplicado por Capone (2009) na simulação de escoamentos de fluidos binghamianos. No entanto, embora os resultados obtidos pelos autores estejam de acordo com comparações teóricas, não há garantias de que as propriedades matemáticas do núcleo de suavização ou seu gradiente sejam obedecidas ao longo da simulação, devido à desorganização no posicionamento das partículas. Neste contexto, há a necessidade de correções no método SPH original.

Takeda et al. (1994) aplicaram um código baseado no SPH ao Poiseuille plano bi e tridimensional, estabelecendo as condições de contorno cinemáticas nas fronteiras através da criação aleatória de partículas externas ao domínio e fixas no espaço. A propositura dos autores garante uma interpolação linear da velocidade entre as partículas real e externa, de tal forma que, na fronteira, a velocidade é nula. Hosseini et al. (2007) simularam escoamentos de fluido newtoniano e não-newtoniano com seu código SPH, representaram a condição de contorno de maneira semelhante à Takeda et al. (1994), com a exceção de que as partículas externas não são geradas aleatoriamente e impõe-se a condição de velocidade nula às partículas externas $(\vec{u}=\overrightarrow{0})$. No contexto computacional, a descrição da condição de contorno por meio da criação dinâmica de partículas em cada intervalo de tempo pode economizar memória, tendo em vista a utilização de um número cada vez maior de partículas nas simulações.

Face ao exposto, este artigo explora o método sem malha SPH renormalizado para simulação de escoamentos laminares, com fluidos newtonianos em superfície livre. A renormalização, Vila (1998), Bonet e Lok (1999) é uma correção que garante que certas propriedades do núcleo de suavização (unicidade) ou seu gradiente (nulidade) sejam obedecidas ao longo da simulação, em todas as partículas fluidas. Aplica-se, adicionalmente, a condição de contorno de partículas fantasma, que cria em cada passo de tempo o número necessário de partículas externas ao domínio.

$\mathrm{O}$ artigo é organizado na apresentação do método SPH e das propriedades da renormalização do núcleo de suavização. O modelo é aplicado para comparação com casos clássicos da literatura, como o Poiseuille plano, Poiseuille com superfície livre e o escoamento de Couette. Adicionalmente, aplica-se a um caso de ruptura de barragem, que possui resultados experimentais.

\section{O MÉTODO SPH}

No SPH, o fluido é tratado como uma série de partículas, com propriedades bem definidas como massa (m), massa específica $(\rho)$, velocidade $(\vec{u})$ e posição $(\vec{r})$. No caso de problemas específicos, pode-se adicionar a energia interna, concentração de um escalar, entre outros.

A transformação do domínio contínuo para o discreto é o cerne do SPH, sendo que para uma função qualquer $\varphi$ (escalar, vetorial ou tensorial), pode-se escrever:

$$
\varphi(\vec{r})=\int \varphi\left(\vec{r}^{\prime}\right) \delta\left(\vec{r}-\vec{r}^{\prime}\right) d \vec{r}^{\prime}
$$

Passando do domínio contínuo para o discreto, tem-se a equação 2 :

$$
\varphi(\vec{r}) \approx \sum_{j} \varphi_{j} W\left(\vec{r}-\vec{r}_{j}, h\right) m_{j} / \rho_{j}
$$

Pelo fato de não existir malha no SPH, não há conectividade entre partículas, tampouco há limitações ao seu movimento, excetuando as restrições de velocidade estabelecidas pela condição CFL (Courant-Friedrichs-Lewy). A interação entre as partículas se dá através da função de suavização $W$, que possui características próprias, como a de tender ao delta de Dirac $(\boldsymbol{\delta})$ quando o seu raio de interação (b) vai para zero.

Uma característica peculiar do SPH é que o gradiente e o divergente de uma grandeza qualquer podem ser expressos pelas equações 3 e 4 :

$$
\begin{aligned}
& \vec{\nabla} \varphi(\vec{r}) \approx \sum_{j} \varphi_{j} \vec{\nabla} W\left(\vec{r}-\vec{r}_{j}, h\right) m_{j} / \rho_{j}, \\
& (\vec{\nabla} \cdot \vec{\varphi}) \approx \frac{1}{\rho} \sum_{j} m_{j}\left(\vec{\varphi}_{j}-\vec{\varphi}\right) \cdot \vec{\nabla} W\left(\vec{r}-\vec{r}_{j}, h\right) .
\end{aligned}
$$


Percebe-se que tanto o gradiente (equação 3) quanto o divergente (equação 4) são obtidos através da aplicação direta do operador nabla à função $W$. Também chamada de kernel, ou núcleo, a função de suavização $W$ tem a responsabilidade de interpolar uma grandeza qualquer entre partículas, limitada pela máxima distância $2 h$ (no caso de funções com suporte compacto). A escolha do núcleo de suavização deve obedecer a certos critérios, como ser normalizada (área sob a curva da função $W$ deve ser unitária) e possuir derivadas primeiras contínuas (de classe $\mathrm{C}^{1}$ ).

É importante também determinar, para cada partícula, as suas partículas vizinhas. Ou seja, para avaliar o termo de somatório nas equações 3 e 4 é preciso estabelecer a lista de vizinhança para todas as partículas. Esse passo demanda grande tempo computacional, e pode ser amenizado utilizando buscas por células e listas de ligação, caso a função $W$ tenha suporte compacto, por exemplo (VASCO et al., 2011).

Aplicando a discretização proposta nas equações 3 e 4 às equações de continuidade e conservação da quantidade de movimento de um fluido ideal e fracamente compressível, adicionando a equação da trajetória, obtém-se o sistema que deve ser resolvido em cada passo de tempo com intervalo $\Delta t$.

$$
\begin{aligned}
& d \vec{r}_{i} / d t=\vec{u}_{i} \\
& d \rho_{i} / d t=\rho_{i} \sum_{j} m_{j} / \rho_{j}\left(\vec{u}_{i}-\vec{u}_{j}\right) \cdot \vec{\nabla}_{i} W_{i j}, \\
& d \vec{u}_{i} / d t=-\sum_{j} m_{j} / \rho_{j}\left(\frac{p_{i}-p_{j}}{\rho_{i} \rho_{j}}\right) \vec{\nabla}_{i} W_{i j}+\vec{F},
\end{aligned}
$$

sendo $p$ a pressão, a força $\vec{F}$ de corpo por unidade de massa e $W_{i j}=W\left(\vec{r}_{i}-\vec{r}_{j}, h\right)$.

Para fechar o sistema de equações 5, 6 e 7 e manter as características explícitas do SPH, utiliza-se uma equação de estado para pressão, como função da massa específica. É utilizada, nesta pesquisa, a expressão dada por equação 8 (LAIGLE et al., 2007):

$$
p_{i}=c^{2}\left(\rho_{i}-\rho\right)
$$

na qual $c$ é a celeridade do som na simulação, escolhida normalmente como $c=10 \mathrm{~V}$, sendo $V$ a máxima velocidade na simulação. Dessa forma, a celeridade do som utilizada na simulação será menor que seu valor físico, no entanto, garantem-se que as variações de massa específica são pequenas (da ordem de $1 \%$ ), evidenciando a característica fracamente compressível do equacionamento (MONAGHAN, 1994; VASCO et al., 2011).

O sistema de equação apresentado (equações 5, 6,7 e 8) vale para um escoamento de fluido ideal com fronteira aberta, e é resolvido usando um método de integração numérica. $\mathrm{Na}$ sequência, detalham-se o sistema de equações para escoamentos de fluido newtoniano e as condições de contorno para fronteiras sólidas.

\section{O SPH PARA ESCOAMENTOS DE FLUIDOS VISCOSOS NEWTONIANOS}

A simulação de escoamentos de fluidos newtonianos passa pelo estabelecimento do laplaciano da velocidade, segundo a filosofia SPH. Embora pareça um problema trivial, uma vez que o gradiente e o divergente já foram definidos (equações 3 e 4, respectivamente), o fato da derivação recair sobre o núcleo de suavização requer atenção especial. Isso se dá devido ao comportamento da derivada segunda do núcleo de suavização, que pode variar o sinal conforme a distância, o que torna a modelagem direta incompatível com a física do fenômeno (MONAGHAN, 2005).

Dessa forma, buscam-se outras maneiras de representar derivadas segundas no SPH. Algumas tentativas foram feitas como, por exemplo, a utilização da própria expressão da viscosidade artificial clássica. A viscosidade artificial é usada em problemas envolvendo escoamentos de fluidos ideais, onde oscilações de pequena magnitude podem surgir, e é inserida como um termo adicional na equação de balanço de quantidade de movimento. A expressão da viscosidade artificial clássica é dada por (MONAGHAN, 1988):

$$
\Pi_{i j}= \begin{cases}-\frac{\left[\alpha\left(c_{i}+c_{j}\right) s_{i j}+\beta \varsigma_{i j}^{2}\right]}{\rho_{i}+\rho_{j}}, & \text { se } \vec{u}_{i j} \cdot \vec{r}_{i j}<0 \\ 0, & \text { se } \vec{u}_{i j} \cdot \vec{r}_{i j} \geq 0\end{cases}
$$

com:

$$
\zeta_{i j}=\frac{\left[\left(h_{i}+h_{j}\right) \vec{u}_{i j} \cdot \vec{r}_{i j}\right]}{\left[r_{i j}^{2}+0,01\left(h_{i}+h_{j}\right)^{2}\right]}
$$

sendo $\alpha$ e $\beta$ constantes, além de ser adotada a notação $\vec{u}_{i j}=\vec{u}_{i}-\vec{u}_{j}$ e $\vec{r}_{i j}=\vec{r}_{i}-\vec{r}_{j}$. A viscosidade artificial (equação 9) é nula para partículas que se afastam umas das outras, o que não corresponde a um comportamento de viscosidade real. Sendo assim, a partir de um rearranjo na equação 9, Monaghan (1997) obteve a equação 11:

$$
\Pi_{i j}=K\left(c_{i}+c_{j}\right) \vec{u}_{i j} \cdot \vec{\jmath} /\left[\left(\rho_{i}+\rho_{j}\right) r_{i j}^{2}\right]
$$

onde $K$ é um parâmetro numérico de viscosidade e $\vec{J}$ um vetor unindo as partículas $i$ e $j$. Destaca-se que $K$ é função tanto dos parâmetros físicos do escoamento como do núcleo de suavização utilizado (Capone, 2009).

Morris et al. (1997) descrevem a derivada segunda combinando uma discretização mista entre SPH e diferenças finitas, sendo que a equação, proposta pelos autores, que aproxima o laplaciano da velocidade, é dada pela equação 12:

$$
\left[\left(\frac{1}{\rho} \vec{\nabla} \cdot \mu \vec{\nabla}\right) \vec{u}\right]_{i}=\sum_{j} \frac{m_{j}\left(\mu_{i}+\mu_{j}\right) \vec{u}_{i j}}{\rho_{i} \rho_{j}}\left(\frac{1}{r_{i j}^{2}} \frac{\partial W_{i j}}{\partial r_{i}}\right)
$$


na qual $\mu$ é a viscosidade dinâmica.

Nesta pesquisa optou-se por uma aproximação bidimensional, adotada por Lachamp (2003). Segundo o autor, a equação de balanço de quantidade de movimento, para uma lei reológica qualquer, é dada por:

$$
\frac{d \vec{u}_{i}^{x}}{d t}=\mathcal{P}_{i j}^{x}+\mathcal{V}_{i j}^{x}+\vec{F},
$$

com:

$$
\mathcal{P}_{i j}^{x}=\sum_{j} m_{j}\left[\left(\sigma_{i}^{x x}+\sigma_{j}^{x x}\right) /\left(\rho_{i} \rho_{j}\right)+\Pi_{i j}\right] \partial W_{i j} / \partial r^{x}
$$

$$
\mathcal{V}_{i j}^{x}=\sum_{j} m_{j}\left(\sigma_{i}^{x y}+\sigma_{j}^{x y}\right) /\left(\rho_{i} \rho_{j}\right) \partial W_{i j} / \partial r^{y},
$$

sendo $x$ e $y$ as direções dos eixos cartesianos, $\mathcal{P}_{i j}^{x}$ a contribuição às forças de contato devido à pressão, $\mathcal{V}_{i j}^{x}$ o termo viscoso e $\sigma_{\mathrm{o}}$ tensor de Cauchy. No caso de escoamentos de fluido newtoniano bidimensionais, foco deste artigo, $\sigma=-I p+2 \mu(D-0,5 \operatorname{tr}(D) I)$, sendo $D$ o tensor taxa de deformação $D=\left(\vec{\nabla} \vec{u}+\vec{\nabla} \vec{u}^{T}\right) / 2$, I a matriz identidade e $\operatorname{tr}(D)$ o traço da matriz $D$.

De posse da equação 13 , tem-se o novo sistema de equações (5, 6, 8 e 13), resolvido nessa pesquisa;

\section{Renormalização do núcleo de suavização}

O núcleo de suavização $W$ deve representar, no domínio discreto, o delta de Dirac. Portanto, espera-se que a função $W$ também apresente as mesmas propriedades do delta de Dirac, como, por exemplo (equação 16):

$$
\int \delta(\vec{r}) d \vec{r}=1
$$

No domínio discreto, a equação 16 assume a forma (equação 17):

$$
\sum_{j} W_{i j} m_{j} / \rho_{j}=1
$$

Considerou-se $W$ uma spline cúbica bidimensional, dada por (equação 18):

$$
W_{i j}=W(b)=\frac{\omega}{h^{2}} \begin{cases}2 / 3-b^{2}+b^{3} / 2, & \text { se } 0<b \leq 1 \\ (2-b)^{3} / 2, & \text { se } 1<b \leq 2 \\ 0, & \text { se } b>2\end{cases}
$$

$\operatorname{com} b=r_{i j}^{2} / h$. A propriedade de área unitária (equação 16) é atendida, para o valor da constante $\omega$ de $10 / 7 \pi$.

Ao longo de uma simulação no SPH, é natural que as partículas, por não possuírem qualquer tipo de interconectividade, apresentem certa desorganização em seu posicionamento, em relação à configuração inicial. Tal fato pode comprometer a estabilidade de uma simulação (MONAGHAN, 2006).

Adicionalmente, não se pode garantir um número constante de vizinhos para cada partícula na simulação. Como o somatório da equação 17 depende da vizinhança da partícula considerada, fica evidente que na proximidade de uma fronteira sólida, a falta de partículas impede que a equação 17 seja satisfeita.

Desta forma, na presença de fronteiras sólidas, procurase completar a vizinhança das partículas próximas à fronteira adicionando partículas fictícias externas ao domínio (TAKEDA et al., 1994; HOSSEINI et al., 2007). No entanto, quando se trata de problemas em superfície livre, tipo ruptura de barragem, medidas diferentes precisam ser adotadas.

A renormalização para Vila (1998), Bonet e Lok (1999) é uma técnica numérica que visa sanar as inconveniências relacionadas ao núcleo de suavização ou ao seu gradiente, dependendo da propriedade escolhida para ser satisfeita. Tomando a equação 17 , pode-se estabelecer um núcleo de suavização corrigido $W_{i p}^{*}$, dado por:

$$
W_{i j}^{*}=W_{i j} /\left(\sum_{k} m_{k} W_{i k} \rho_{k}^{-1}\right) .
$$

A equação 19 é conhecida como o interpolador de Shepard (1968) e pode-se notar que, independente do número de vizinhos, a equação 17 é sempre obedecida. No entanto, a equação 19 é exata apenas quando interpola funções constantes.

Para interpolar exatamente polinômios de ordem $N$, aplica-se o núcleo corrigido pelo interpolador MLS (Moving Least Squares, Dilts, 1999). Entretanto, por causa do número crescente de operações e tempo computacional, limita-se a técnica à ordem $N=1$.

A aplicação de um interpolador MLS de ordem maior que zero requer a solução de um sistema linear, em que a matriz dos coeficientes pode ser singular devido à falta de vizinhos. Neste caso, faz-se uma verificação da possibilidade de redução da matriz, para uma submatriz com determinante não nulo. Esta propriedade de envelopamento da matriz dos coeficientes constitui uma das características do interpolador MLS (DILTS, 1999). Cabe lembrar que, caso $N=0, \mathrm{o}$ MLS reverte à correção de Shepard (equação 19).

Finalmente, a reinicialização da massa específica é uma renormalização do núcleo de suavização, que tem por objetivo amenizar o fato da equação da continuidade não conservar exatamente o volume. A correção envolve aplicar, em intervalos de tempo pré-definidos: $\rho_{i}=\Sigma_{j} m_{j} W_{i j}^{*}$, (LAIGLE et al., 2007).

\section{RENORMALIZAÇÃO DO GRADIENTE DO NÚCLEO DE SUAYVIZAÇÃO}

Uma das correções do gradiente do núcleo de suavização visa resolver a equação 20 (BONET; LOK, 1999; COLAGROSSI, 2004):

$$
\sum_{j} r_{j} \otimes \nabla_{i} W_{i j} m_{j} / \rho_{j}=I,
$$

sendo $\otimes$ o produto tensorial entre dois vetores.

Para satisfazer a equação 20, basta modificar o gradiente do núcleo, de tal forma que: 


$$
\left(\nabla_{i} W_{i j}\right)^{*}=B_{i j} \nabla_{i} W_{i j}
$$

onde $\left(\nabla_{i} W_{i j}\right)^{*}$ é o gradiente do núcleo corrigido e $B_{i j}$ a matriz de renormalização. Desta forma, se $B_{i j}$ for dada pela equação 22:

$$
B_{i j}=\left(\sum_{j} r_{j} \otimes \nabla_{i} W_{i j} m_{j} / \rho_{j}\right)^{-1},
$$

garante-se que a restrição imposta pela equação 20 sempre será satisfeita, independente das posições das partículas.

As correções aplicadas às simulações empreendidas na pesquisa englobam, portanto, a renormalização do núcleo de suavização (reinicialização da massa específica) e do gradiente.

\section{Condições de Contorno}

As condições de contorno para escoamentos de fluido newtoniano, nas fronteiras sólidas do domínio, são impostas através das partículas fantasma. Nesta técnica, quando uma partícula aproxima-se da fronteira, há o espelhamento da partícula, criando uma partícula externa ao domínio, com a mesma distância normal à fronteira. A partícula externa assim criada, ou partícula fantasma, possui as mesmas características da partícula real (massa, por exemplo). Entretanto, parâmetros como o sentido do vetor velocidade ou o valor da pressão são atribuídos de acordo com a condição de contorno da fronteira. Tal procedimento mantém o número de vizinhos das partículas em um valor aproximadamente constante, evitando possíveis instabilidades numéricas nesta região (LACHAMP, 2003).

Em relação à velocidade das partículas fantasma, a condição de parede pode ser dada por $\vec{u}_{g} \cdot \vec{n}=-\vec{u}_{i} \cdot \vec{n}$ sendo o versor $\vec{n}$ normal à fronteira. Para a velocidade tangencial, podem-se elencar três formas diferentes (equações 23, 24 e 25) para representar as condições de contorno (LACHAMP, 2003; SOUTO-IGLESIAS et al., 2010; COLAGROSSI, 2004):

$$
\begin{aligned}
& \vec{u}_{g} \cdot \vec{s}=-\vec{u}_{i} \cdot \vec{s} \\
& \vec{u}_{g} \cdot \vec{s}=\vec{u}_{i} \cdot \vec{s} \\
& \vec{u}_{g} \cdot \vec{s}=0
\end{aligned}
$$

na qual o subíndice $i$ representa a partícula fluida, $g$ representa a partícula fantasma correspondente e $\vec{S}$ é o versor tangencial.

$\mathrm{Na}$ equação 23, a velocidade tangencial da partícula fantasma correspondente é igual e oposta à da partícula fluida. Esta seria a melhor tradução da condição de contorno do tipo aderência ou não deslizamento. A equação 24 considera aspectos de simetria, ou espelhamento das propriedades. Esta condição de contorno representa bem a condição de deslizamento.

Enquanto a equação 25 representa a velocidade tangencial das partículas fantasma nula, qualquer que seja $i$. Embora não exista equivalência matemática para esse conceito, pode-se considerar que o efeito que essa imposição causa ao escoamento é o da aderência.

Há outras maneiras para incorporar a condição de contorno de aderência para escoamentos de fluido newtoniano. Maciá et al. (2011) testaram diferentes equações para as condições de contorno de aderência através do cálculo do laplaciano da velocidade na fronteira, para o problema do Poiseuille plano, com o SPH. Os autores mostraram que nenhuma técnica atualmente utilizada na literatura reproduz o resultado teórico esperado, podendo inclusive contribuir para geração de instabilidades.

Foram utilizados nesta pesquisa, as partículas fantasma com condição de parede para velocidade normal à fronteira $\left(\vec{u}_{g} \cdot \vec{n}=-\vec{u}_{i} \cdot \vec{n}\right)$ e condição de aderência para velocidade tangencial. Para as demais grandezas (pressão, massa específica, viscosidade dinâmica, etc.), adota-se a condição de contorno do tipo Neumann: $\partial \varphi / \partial \vec{n}=0$. Para o caso de superfície livre, no fundo do canal, tem-se: $\partial p / \partial \vec{n}=\rho \vec{F} \cdot \vec{n}$. Assim, garante-se que o gradiente de pressão é calculado corretamente (LACHAMP, 2003). As fronteiras sólidas são perfeitamente elásticas (RODRIGUEZ-PAZ; BONET, 2004).

\section{RESULTADOS}

Inicialmente, faz-se um teste para se verificar a capacidade de reprodução de um escoamento estacionário com a utilização das fronteiras periódicas. Posteriormente, aspectos estacionários e transientes de casos clássicos são testados, como o Poiseuille com superfície livre, o Poiseuille plano e o escoamento de Couette. Finalmente, comparam-se os resultados numéricos e experimentais (Leite, 2009) para a evolução do alcance da frente em um problema do tipo ruptura de barragem.

Os parâmetros numéricos comuns a todas as simulações realizadas nesta pesquisa estão destacados na Tabela 1 .

Tabela 1 - Parâmetros numéricos nas simulações

\begin{tabular}{|l|l|}
\hline Compr. de suavização $(b)$ & $1,2 \Delta x$ \\
\hline Núcleo de suavização $(W)$ & spline cúbica 2D (eq. 18) \\
\hline Integrador numérico & Runge-Kutta de $2^{\mathrm{a}}$ ordem \\
\hline Passo de tempo $(\Delta t)$ & $\min \left(\left(0,5 h_{i}\right) /\left(u_{i}+c_{i}\right) ;\right.$ \\
& $\left.0,1736 h_{i}^{2} \rho_{i} / \mu\right)$ \\
\hline
\end{tabular}

\section{Fronteiras periódicas}

Neste tipo especial de condição de contorno, as partículas fluidas que abandonam o domínio são automaticamente reintroduzidas, em outra região, de modo que seja possível alcançar, ao cabo de tempo finito, um regime de escoamento estacionário.

De modo a testar a fronteira periódica em uma simulação, propõe-se um problema similar àquele apresentado por Monaghan (2006). Trata-se de um escoamento em um domínio retangular $(1,0 \mathrm{~m} \times 0,6 \mathrm{~m})$, com ausência de forças externas e com um campo de velocidade com componentes nas direções $x$ e $y$ dadas por: $u_{x}(y)=2 \pi \operatorname{sen}(y / 0,6)$ e $u_{y}(y)=0$, respectivamente (em $\mathrm{m} / \mathrm{s})$.

As condições de contorno são periódicas tanto nas 
fronteiras verticais quanto nas horizontais. O núcleo de suavização usado é o spline cúbico bidimensional normalizado (equação 18). O comprimento de suavização adotado vale $h=1,2 \Delta x$, Monaghan (1994), Morris et al, (1997), Lachamp (2003), sendo $\Delta x$ o espaçamento inicial entre as partículas. A resolução espacial escolhida foi de $\Delta x \approx 0,017 \mathrm{~m}$, o que resultou em um número de partículas pouco maior que 2100 .

Como condição inicial, as partículas são posicionadas nos vértices formados por quadrados, de lado $\Delta x$. A massa específica inicial das partículas $\rho_{i}=\rho=1000 \mathrm{~kg} / \mathrm{m}^{3}$, o que resulta em $p_{i}=0$ (conforme a equação 8 ). O fluido possui uma viscosidade cinemática $v=10^{-3} \mathrm{~m}^{2} / \mathrm{s}$, caracterizando escoamento com número de Reynolds de 600. É aplicada, a cada 0,01 s, a reinicialização da massa específica baseada no método MLS. Emprega-se, também, a renormalização do gradiente do núcleo de suavização, como medida adicional de correção. A celeridade do som, na simulação, vale $23,29 \mathrm{~m} / \mathrm{s}$.

O passo de tempo $\Delta t$ é calculado automaticamente a cada iteração, aproveitando da característica explícita do SPH, e é o menor valor entre $\Delta t_{1}$ e $\Delta t_{2}$, sendo $\Delta t_{1}=0,5 h_{i} /\left(u_{i}+c\right)$ e $\Delta t_{2}=0,1736 h_{i}^{2} \rho_{i} / \mu$, calculado para todas as partículas fluidas. O integrador numérico utilizado foi do tipo Runge-Kutta de $2^{a}$ ordem, que apresenta um bom compromisso entre tempo computacional e erro numérico (MONAGHAN, 2005). A Tabela 2 resume os parâmetros adotados na simulação.

A Figura 1 apresenta a distribuição de velocidade em $t=1,5 \mathrm{~s}$. A fronteira periódica foi bem reproduzida, uma vez que o perfil de velocidades se manteve inalterado (erros relativos máximos inferiores a 1\%). Tal comportamento também foi obtido por Monaghan (2006), com exceção aos pontos onde a derivada $\left|\partial^{2} u_{x} / \partial y^{2}\right|$ é grande. Na região apontada, os resultados do referido autor apresentam uma ligeira redução do campo de velocidade. Ademais, mesmo com a presença da viscosidade, as partículas se mantêm organizadas, fato esse ilustrado pelos pontos na Figura 1, que representam uma linha de partículas, que permanecem com a mesma altura do começo da simulação.

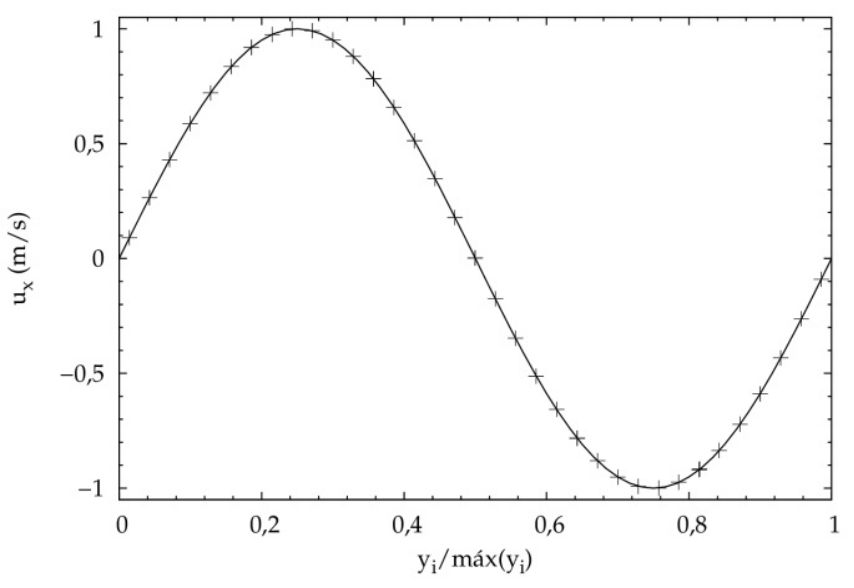

Figura 1 - Comparação entre o perfil de velocidades esperado (linha contínua, teórica) com o numérico $(+)$, em $t=1,5 \mathrm{~s}$
Tabela 2 - Dados da simulação para o caso testes da fronteira periódica

\begin{tabular}{|l|l|}
\hline \multicolumn{2}{|c|}{ 1- Parâmetros geométricos } \\
\hline \multicolumn{2}{|c|}{ 1.1- Dimensões características } \\
\hline Horizontal Parâmetros físicos \\
\hline Vertical & $1,0 \mathrm{~m}$ \\
\hline \multicolumn{2}{|c|}{ 3- Parâmetros numéricos } \\
\hline Massa específica $(\rho)$ & $1000 \mathrm{~kg} / \mathrm{m}^{3}$ \\
\hline Viscosidade cinemática $(v)$ & $10^{-3} \mathrm{~m}^{2} / \mathrm{s}$ \\
\hline \multicolumn{2}{|c|}{ 3.1 -Condições iniciais } \\
\hline Espaçamento médio $(\Delta x)$ & $0,017 \mathrm{~m}$ \\
\hline Celeridade do som & $\vec{u}=(2 \pi$ sen $(y / 0,6) ; 0)$ \\
\hline Reinicialização de $\rho$ & $\rho_{i}=\rho$ \\
\hline \multicolumn{2}{|c|}{$\rho$ ) } \\
\hline Cinemática $3.2-$ Condições de contorno \\
\hline Dinâmica & Em $x=0$ e $x=1$ \\
\hline \multicolumn{2}{|c|}{ 3 cada $0,01 \mathrm{~s}$} \\
\hline Periodicidade (horizontal) & Em $y=0$ e $y=0,6$ \\
\hline Periodicidade (vertical)
\end{tabular}

$\mathrm{Na}$ Figura 2, apresenta-se a posição das partículas, que permanecem organizadas, pois continuam posicionadas nos vértices de quadrados de lado $\Delta x$. Tal fato indica sucesso na representação da condição de contorno periódica. Destaca-se também que não há variação substancial na massa específica, que consequentemente ilustra pouca variação do campo de pressões (uma vez que $p \rightarrow p(\rho)$, vide equação 8 ).

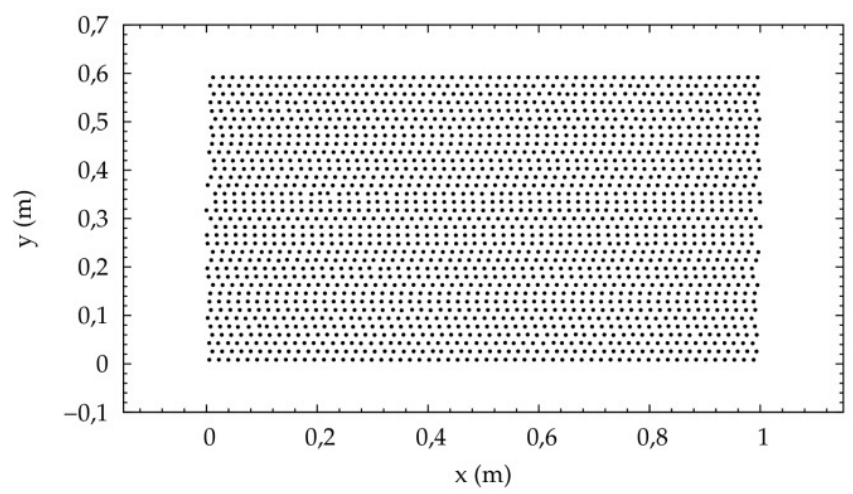

Figura 2 - Posição das partículas em $t=1,5 \mathrm{~s}$

\section{Poiseuille com superfície livre}

Escoamentos com superfície livre, da Hidráulica de canais abertos, podem ser representados pelo clássico problema da Mecânica dos Fluidos, qual seja, Poiseuille plano - escoamento entre placas paralelas com forçante sem a presença da placa superior. Tal configuração caracteriza, portanto, um escoamento cuja força motriz é a gravidade. Lachamp (2003) apresenta a solução $u_{x}(y)$, em regime permanente, dada pela equação 26 :

$$
u_{x}(y)=\frac{\rho g d^{2} \operatorname{sen}(\theta)}{\mu}\left[\frac{y}{d}-\frac{1}{2}\left(\frac{y}{d}\right)^{2}\right]
$$


sendo $d$ a lâmina d'água normal e $\theta$ o ângulo de inclinação do canal em relação à horizontal.

De modo a encontrar uma resolução adequada, foram escolhidas quatro configurações distintas para a razão entre a altura da lâmina d'água e o espaçamento inicial $(d / \Delta x): 25,35$, 40 e 50 . A celeridade do som da simulação vale $c=23,29 \mathrm{~m} / \mathrm{s}$.

A condição de contorno de aderência é representada pelas partículas fantasma, utilizando tanto a velocidade tangencial nula $\left(\vec{u}_{g} \cdot \vec{s}=0\right)$ quanto a velocidade oposta $\left(\vec{u}_{g} \cdot \vec{s}=-\vec{u}_{i} \cdot \vec{s}\right)$. Como condição inicial, as partículas são dispostas em quadrados, de lado $\Delta x$, com velocidade nula, o que resulta em um número de partículas de 1065 a 4232.

Aplica-se, a cada $0,01 \mathrm{~s}$, a interpolação baseada no método MLS. Tal correção é realizada, neste caso, tanto à massa específica quanto à velocidade. É necessária a aplicação da interpolação MLS ao campo de velocidade tendo em vista oscilações naturais apresentadas pela formulação adotada (CUEILLE, 2005).

Os parâmetros físicos e geométricos do Poiseuille com superfície livre e um resumo geral das considerações efetuadas são apresentadas na Tabela 3. Já os resultados, ilustrando o comportamento da simulação com a variação do número de partículas e o efeito da velocidade da partícula fantasma no perfil de velocidades estacionário, podem ser vistos na Figura 3.

Tabela 3 - Dados da simulação para o Poiseuille com superfície livre

\section{1- Parâmetros geométricos}

\begin{tabular}{|l|l|}
\hline \multicolumn{2}{|c|}{ 1- Parâmetros geométricos } \\
\hline \multicolumn{2}{|c|}{ 1.1- Dimensões características } \\
\hline Horizontal & $1,0 \mathrm{~m}$ \\
\hline Vertical $(d)$ & $0,6 \mathrm{~m}$ \\
\hline Inclinação do canal $(\theta)$ & $16,45^{\circ}$ \\
\hline \multicolumn{2}{|c|}{ 2- Parâmetros físicos } \\
\hline Massa específica $(\rho)$ & $1000 \mathrm{~kg} / \mathrm{m}^{3}$ \\
\hline Viscosidade cinemática $(v)$ & $1 \mathrm{~m}^{2} / \mathrm{s}$ \\
\hline \multicolumn{2}{|c|}{ 3- Parâmetros numéricos } \\
\hline Espaçamento médio $(\Delta x)$ & 0,024 a $0,012 \mathrm{~m}$ \\
\hline Celeridade do som & $23,29 \mathrm{~m} / \mathrm{s}$ \\
\hline Reinicialização de $\rho$ & $\mathrm{A}$ cada $0,01 \mathrm{~s}$ \\
\hline \multicolumn{2}{|c|}{ 3.1 -Condições iniciais } \\
\hline Cinemática & Campo de velocidadenulo \\
\hline Dinâmica & $\rho_{i}=\rho$ \\
\hline \multicolumn{2}{|c|}{ 3.2- Condições de contorno } \\
\hline Parede $(y=0)$ & $\vec{u}_{g} \cdot \vec{n}=-\vec{u}_{i} \cdot \vec{n}$ \\
\hline Aderência $(y=0)$ & $\vec{u}_{g} \cdot \vec{s}=0$ ou \\
& $\vec{u}_{g} \cdot \vec{s}=-\vec{u}_{i} \cdot \vec{s}$ \\
\hline
\end{tabular}

A Figura 3a sugere que o perfil dado pela condição de aderência $\vec{u}_{g} \cdot \vec{s}=0$. apresenta melhores resultados na parte superior do perfil de velocidades, em enquanto a condição de contorno $\vec{u}_{g} \cdot \vec{t}=-\vec{u}_{i} \cdot \vec{s}$ representa melhor a metade inferior do perfil de velocidades.

$\mathrm{Na}$ Figura 3b, utilizando como condição de aderência, mesmo para a menor razão $d / \Delta x$, o perfil de velocidade numérico não apresenta diferença significativa do perfil teórico. Tal fato é comprovado pelo erro relativo da velocidade máxima de pouco mais de $2 \%$.
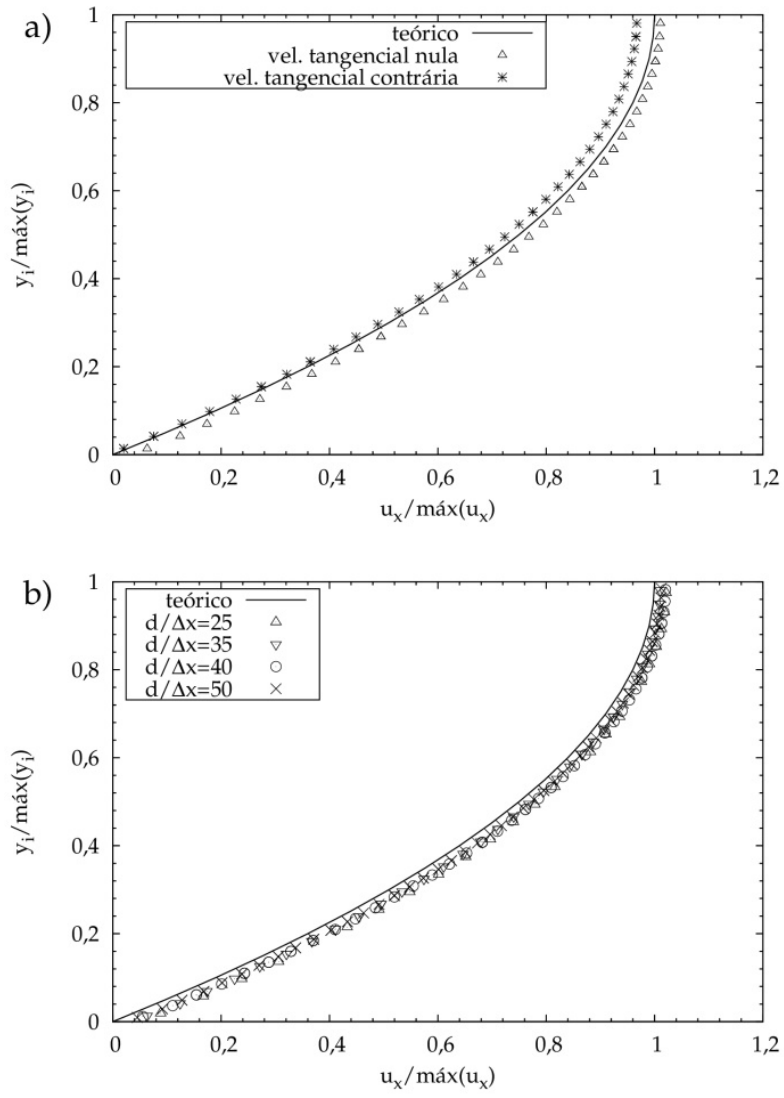

Figura 3 - Estudo paramétrico: a) influência do tipo de condição de contorno utilizada no perfil de velocidade estacionário, para $d / \Delta x=35$ e $b$ ) testes com vários números de partículas para determinar a razão $d / \Delta x$ ideal

\section{Poiseuille plano}

O Poiseuille plano é um escoamento que se dá entre placas paralelas, consideradas infinitas, com uma forçante (gradiente de pressão). Nesta simulação, utiliza-se como forçante uma força de corpo por unidade de massa $\left(F_{\nu}\right)$, paralela às placas. A solução teórica transiente $u_{x}(y, t)$ desenvolvida em termos de séries é dada por (Morris et al, 1997):

$$
u_{x}(y, t)=\frac{F_{x}}{2 v} y(\varepsilon-y)-T_{p}
$$

com:

$$
T_{p}=\sum_{k=0}^{\infty} T_{p 1} T_{p 2} T_{p 3},
$$

sendo $\mathcal{E}$ a distância entre as placas e $T_{p 1}, T_{p 2}$ e $T_{p 3}$ dados por:

$$
\begin{aligned}
T_{p 1} & =\frac{4 F_{x} \varepsilon^{2}}{v \pi^{3}(2 k+1)^{3}}, \\
T_{p 2} & =\operatorname{sen}\left(\frac{k y(2 k+1)}{\varepsilon}\right),
\end{aligned}
$$




$$
T_{p 3}=\exp \left(-\frac{(2 k+1)^{2} \pi^{2} v}{\varepsilon^{2}} t\right)
$$

Os dados para a simulação numérica do Poiseuille plano foram resumidos na Tabela 4. Já os perfis de velocidade na parte central do domínio $(x=0,5 \mathrm{~m})$, no regime permanente, podem ser vistos na Figura 4.

Tabela 4 - Dados da simulação para o Poiseuille plano

\begin{tabular}{|l|l|}
\hline \multicolumn{2}{|c|}{ 1- Parâmetros geométricos } \\
\hline \multicolumn{2}{|c|}{ 1.1- Dimensões características } \\
\hline Horizontal & $1,0 \mathrm{~m}$ \\
\hline Vertical $(\varepsilon)$ & $0,6 \mathrm{~m}$ \\
\hline F. corpo por massa $(F x)$ & $2,78 \mathrm{~m} / \mathrm{s}^{2}$ \\
\hline \multicolumn{2}{|c|}{ 2- Parâmetros físicos } \\
\hline Massa específica $(\rho)$ & $1000 \mathrm{~kg} / \mathrm{m}^{3}$ \\
\hline Viscosidade cinemática $(v)$ & $1 \mathrm{~m}^{2} / \mathrm{s}$ \\
\hline \multicolumn{2}{|c|}{ 3- Parâmetros numéricos } \\
\hline Espaçamento médio $(\Delta x)$ & $0,017 \mathrm{~m}$ \\
\hline Celeridade do som & $23,29 \mathrm{~m} / \mathrm{s}$ \\
\hline Reinicialização de $\rho$ & A cada $20 \Delta t$ \\
\hline \multicolumn{2}{|c|}{3.1 -Condições iniciais } \\
\hline Cinemática & Campo de velocidadenulo \\
\hline Dinâmica & $\rho_{i}=1,01 \rho$ \\
\hline \multicolumn{2}{|c|}{ 3.2- Condições de contorno } \\
\hline Aderência $(y=0$ e $y=0,6)$ & $\vec{u}_{g} \cdot \vec{s}=0$ \\
\hline Periodicidade (horizontal) & Em $x=0 \mathrm{e} x=1$ \\
\hline
\end{tabular}

Pode-se perceber, pelos resultados observados na Figura 4, que o modelo SPH proposto é capaz de reproduzir o escoamento transiente em regime laminar, uma vez que o erro relativo máximo no perfil de velocidades é inferior a $1 \%$.

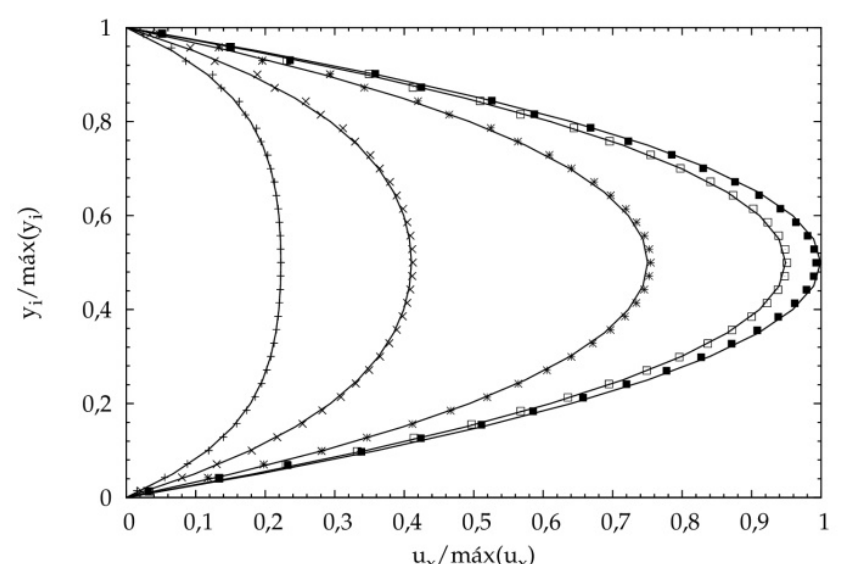

Figura 4 - Resultados analíticos e numéricos do perfil de velocidade no centro do domínio computacional, nos tempos $t=0,01 \mathrm{~s}(+)$, $0,02 \mathrm{~s}(\times), 0,05 \mathrm{~s}(*), 0,1 \mathrm{~s}(\square)$ e $\infty$ (匹). Os símbolos indicam os resultados numéricos, enquanto que a linha contínua representa os resultados analíticos, nos respectivos tempos

\section{Escoamento de Couette}

O escoamento de Couette é o escoamento entre duas placas paralelas, também consideradas infinitas, sendo que uma das placas possui velocidade não nula. Na simulação SPH, a placa superior é dotada de velocidade, cuja intensidade $V_{p s}$ é de $0,1 \mathrm{~m} / \mathrm{s}$, deslocando-se da esquerda para a direita. A componente horizontal da solução teórica transiente $u_{x}(y, t)$ do problema é dada por (MORRIS et al., 1997):

$$
u_{x}(y, t)=V_{p s} y / \varepsilon+T_{c},
$$

com:

$$
T_{c}=\sum_{k=0}^{\infty} \frac{2 V_{p s}}{k \pi}(-1)^{k} \operatorname{sen}\left(\frac{k \pi y}{\varepsilon}\right) \exp \left(-\frac{v k^{2} \pi^{2}}{\varepsilon^{2}} t\right) .
$$

Para o escoamento de Couette, são mantidos os mesmos parâmetros do Poiseuille plano, com as seguintes alterações: a condição de contorno na fronteira superior passa a ser $\vec{u}_{g}=\left(V_{p s} ; 0\right)$ e remove-se a força de corpo. Tais parâmetros numéricos são resumidos na Tabela 5 .

A comparação do perfil de velocidade analítico e numérico, na parte central do domínio $(x=0,5 \mathrm{~m})$, pode ser vista na Figura 5. Observa-se que tanto o comportamento geral quanto o aspecto transiente do escoamento de Couette são reproduzidos no código SPH. Adicionalmente, para o tempo 0,01 s, ocorre uma oscilação dos resultados numéricos, para $u_{x} / \max \left(u_{x}\right)>0,2$,

\begin{tabular}{|c|c|}
\hline \multicolumn{2}{|c|}{ 1- Parâmetros geométricos } \\
\hline \multicolumn{2}{|c|}{ 1.1- Dimensões características } \\
\hline Horizontal & $1,0 \mathrm{~m}$ \\
\hline Vertical $(\varepsilon)$ & $0,6 \mathrm{~m}$ \\
\hline \multicolumn{2}{|c|}{ 2- Parâmetros físicos } \\
\hline Massa específica $(\rho)$ & $1000 \mathrm{~kg} / \mathrm{m}^{3}$ \\
\hline Viscosidade cinemática $(v)$ & $1 \mathrm{~m}^{2} / \mathrm{s}$ \\
\hline \multicolumn{2}{|c|}{ 3- Parâmetros numéricos } \\
\hline Espaçamento médio $(\Delta x)$ & $0,017 \mathrm{~m}$ \\
\hline Compr. de suavização $(b)$ & $1,2 \Delta x$ \\
\hline Núcleo de suavização $(W)$ & spline cúbica 2D (eq. 18) \\
\hline Celeridade do som & $23,29 \mathrm{~m} / \mathrm{s}$ \\
\hline Integrador numérico & Runge-Kutta de $2^{\mathrm{a}}$ ordem \\
\hline Passo de tempo $(\Delta t)$ & $\begin{array}{l}\min \left(\left(0,5 h_{i}\right) /\left(u_{i}+c_{i}\right)\right. \\
\left.0,1736 h_{i}^{2} \rho_{i} / \mu\right)\end{array}$ \\
\hline Reinicialização de $\rho$ & A cada $20 \Delta t$ \\
\hline \multicolumn{2}{|c|}{3.1 -Condições iniciais } \\
\hline Cinemática & Campo de velocidadenulo \\
\hline Dinâmica & $\rho_{i}=1,01 \rho$ \\
\hline \multicolumn{2}{|c|}{ 3.2- Condições de contorno } \\
\hline Cinemática (para $y=0,6$ ) & $\vec{u}_{g}=(0,1 ; 0)(\mathrm{m} / \mathrm{s})$ \\
\hline Aderência $(y=0$ e $y=0,6)$ & $\vec{u}_{g} \cdot \vec{s}=0$ \\
\hline Periodicidade (horizontal) & $\operatorname{Em} x=0$ e $x=1$ \\
\hline
\end{tabular}
mesmo depois de uma suavização no campo de velocidades.

Tabela 5 - Dados da simulação para o escoamento de Couette 


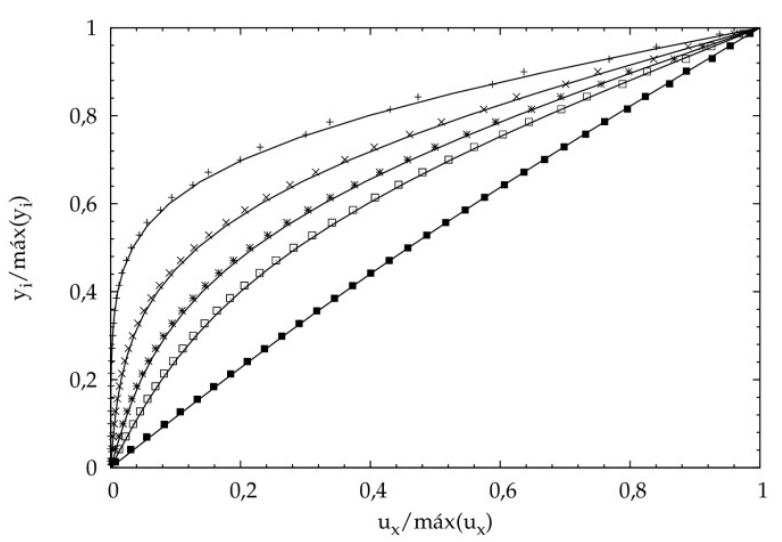

Figura 5 - Resultados analíticos e numéricos do perfil de velocidade no centro do domínio computacional, nos tempos $t=0,01 \mathrm{~s}(+)$, 0,02 s (×), 0,03 s (*), 0,04 s (口) e $\infty$ (匹). Os símbolos indicam os resultados numéricos, enquanto que a linha contínua representa os resultados analíticos, nos respectivos tempos

Tal fato ilustra as oscilações naturais em torno da solução, que são inerentes à equação de conservação de quantidade de movimento adotada.

\section{Problema tipo ruptura de barragem}

Rupturas de barragem são problemas que vêm sendo estudados desde o final do século XIX, com um dos trabalhos pioneiros de Ritter (1892), que considerou o escoamento de fluido ideal. Posteriormente, para levar em conta os aspectos viscosos do escoamento, o efeito do atrito de fundo (proporcional ao coeficiente de Chézy) foi adicionado. O sistema de equações resultante, com a hipótese de águas rasas, tem sido resolvido com o auxílio de métodos numéricos tradicionais, que utilizam malha. Recentemente, procura-se também aplicar os MSM em problemas do tipo ruptura de barragens em fluidos newtonianos (HOSSEINI et al., 2007).

Aplica-se o código SPH desenvolvido ao problema tipo ruptura de barragens em fluido newtoniano, considerando os parâmetros numéricos apresentados na Tabela 1. Neste caso, as correções desempenham papel importante, tendo em vista que as partículas apresentam desorganização natural em relação ao seu posicionamento inicial, ao longo da simulação.

Para comparação com a simulação numérica, foram utilizados os resultados experimentais de Leite (2009), reali-

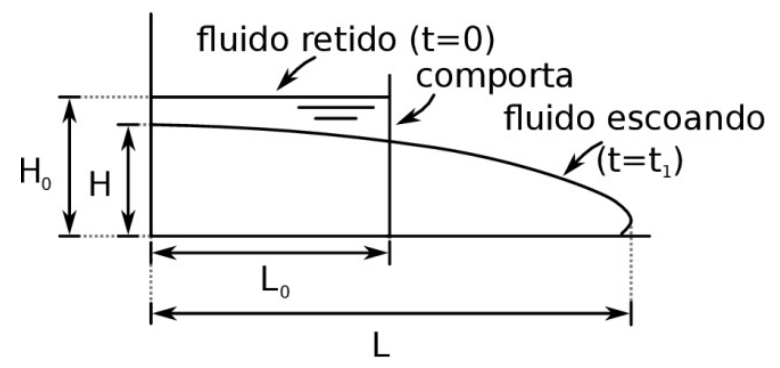

Figura 6 - Croqui esquemático para o problema tipo ruptura de barragem zados em um reservatório de 0,5 $\mathrm{m}$ de largura por $0,3 \mathrm{~m}$ de profundidade, variando a altura do fluido retido $H_{0}$ entre 0,08 e $0,12 \mathrm{~m}$. O fluido é composto por uma solução de glicerol, $\operatorname{com} \rho=1262 \mathrm{~kg} / \mathrm{m}^{3}$ e $\mu=0,86 \mathrm{~Pa}$ s. A Figura 6 ilustra um esquema do fenômeno, evidenciando o comportamento da frente de avanço após o início do movimento.

Nesta simulação, a lei de estado para a pressão (equação 8) é substituída pela equação 34, melhor adaptada a problemas envolvendo descontinuidades (MONAGHAN, 1994; MONAGHAN, 2005).

$$
p_{i}=\frac{c^{2} \rho_{0}}{7}\left(\left(\frac{\rho_{i}}{\rho_{0}}\right)^{7}-1\right)
$$

As partículas são dispostas inicialmente nos vértices de quadrados de lado $\Delta x$, obedecendo à resolução vertical de $H_{0} / \Delta x=35$, o que resulta em 5753 partículas. A condição inicial dinâmica e cinemática é distribuição hidrostática de pressão e velocidade nula, respectivamente. A condição de contorno cinemática nas fronteiras é de parede e aderência, com velocidade tangencial contrária $\left(\vec{u}_{g} \cdot \vec{s}=-\vec{u}_{i} \cdot \vec{s}\right)$. Foram aplicadas a renormalização do gradiente do núcleo de suavização e a reinicialização da massa específica, a cada $30 \Delta t$. A celeridade do som na simulação vale 12,6, 14,0 e 15,6 m/s, para as lâminas iniciais de $0,08,0,10$ e $0,12 \mathrm{~m}$, respectivamente. A Tabela 6 apresenta os demais parâmetros adotados.

Tabela 6 - Dados da simulação para o problema tipo ruptura de barragem

\begin{tabular}{|l|l|}
\hline \multicolumn{2}{|c|}{ 1- Parâmetros geométricos } \\
\hline \multicolumn{2}{|c|}{ 1.1- Dimensões características } \\
\hline Altura do fluido retido & $0,08,0,10$ e $0,12 \mathrm{~m}$ \\
\hline Largura do reservatório & $0,5 \mathrm{~m}$ \\
\hline \multicolumn{2}{|c|}{ 2- Parâmetros físicos } \\
\hline Massa específica $(\rho)$ & $1262 \mathrm{~kg} / \mathrm{m}^{3}$ \\
\hline Viscosidade dinâmica $(\boldsymbol{\mu})$ & $0,86 \mathrm{~Pa} \mathrm{~s}$ \\
\hline \multicolumn{2}{|c|}{ 3- Parâmetros numéricos } \\
\hline Espaçamento médio $(\Delta x)$ & 0,0023 a $0,0034 \mathrm{~m}$ \\
\hline Celeridade do som & $12,6,14,0$ e $15,6 \mathrm{~m} / \mathrm{s}$ \\
\hline Reinicialização de $\rho$ ? & A cada $30 \Delta t$ \\
\hline \multicolumn{2}{|c|}{ 3.1 -Condições iniciais } \\
\hline Cinemática & Campo de velocidadenulo \\
\hline Dinâmica & Perfil hidrostático \\
\hline \multicolumn{2}{|c|}{ 3.2- Condições de contorno } \\
\hline Parede (y $=0)$ & $\vec{u}_{g} \cdot \vec{n}=-\vec{u}_{i} \cdot \vec{n}$ \\
\hline Aderência (y $=0)$ & $\vec{u}_{g} \cdot \vec{s}=-\vec{u}_{i} \cdot \vec{s}$ \\
\hline
\end{tabular}

Os resultados para o avanço da frente, para as distintas alturas de represamento, são apresentados nas Figuras 7a, 7b e 7c.

Primeiramente, observa-se um distanciamento entre os resultados experimentais e numéricos da solução teórica de Ritter (1892). Esta diferença é causada pela característica essencialmente inercial da formulação de Ritter (1892), abandonando a contribuição de aspectos viscosos em seu modelo. 

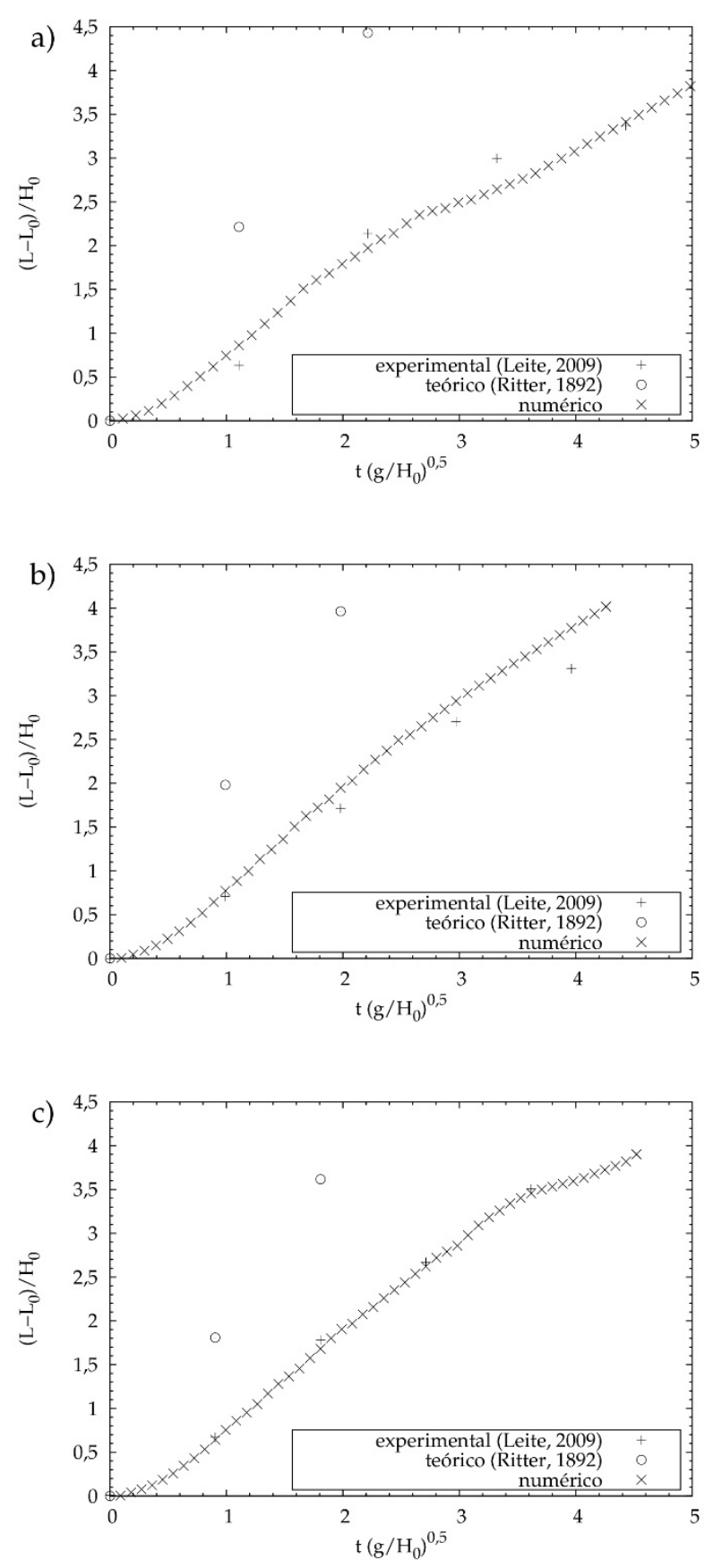

Figura 7 - Comparação entre resultados experimentais de Leite (2009), teóricos de Ritter (1982) e numéricos para o problema de ruptura de barragem retendo material newtoniano, com altura de ruptura: a) $H_{0}=0,08 \mathrm{~m}$ e b) $H_{0}=0,1 \mathrm{~m} \mathrm{e} \mathrm{c)} H_{0}=0,12 \mathrm{~m}$

O resultado numérico mostra uma mudança na inclinação do alcance da frente, notado nos pontos $(2,8 ; 2,5)$ da Figura 7 a e $(3,6 ; 3,5)$ da Figura 7 c. Este fato pode ser atribuído à maneira como o alcance da frente é interpretado no SPH. A frente de ruptura é composta por diversas partículas, e a posição daquela mais distante, em um determinado tempo, fornece o alcance da frente. No entanto, devido ao ângulo de contato entre o fluido escoante e o fundo (Figura 6), às vezes, há uma mudança repentina na posição das partículas, devido ao elevado gradiente de pressão que se forma nesta região.

Outro fator a ser destacado é a forma como a condição de contorno de aderência no fundo é escrita no SPH. Maciá et al. (2011) estudaram diferentes maneiras de impor a condição de aderência em escoamentos viscosos e concluíram que nenhuma técnica que figura na literatura consegue reproduzir teoricamente tal condição. Além disso, os autores mostram que a forma do perfil de velocidades resultante depende do comprimento de suavização adotado na simulação. Estes dois fatores, aliados à maneira de encontrar o alcance da frente da onda, podem ter contribuído para as diferenças entre os resultados numéricos e experimentais. Os erros relativos variaram entre os limites de 0,7 a $14 \%$, com a exceção de um único valor de $34 \%$, correspondendo ao ponto $(1,05 ; 0,6)$ na Figura 7 a. Mesmo considerando este ponto, o erro relativo médio ficou abaixo dos $10 \%(\sim 9,5 \%)$, margem considerada aceitável em problemas de Engenharia.

A Figura 8 traz um instante da simulação numérica, mostrando a criação das partículas fantasma dinamicamente no fundo do canal. Além disso, nota-se que a forma da frente de ruptura é compatível com observações experimentais e é ocasionada pela condição de aderência no fundo e a distribuição de velocidades é compatível com a transformação de energia (carga de pressão para cinética) nas proximidades da ruptura da barragem.
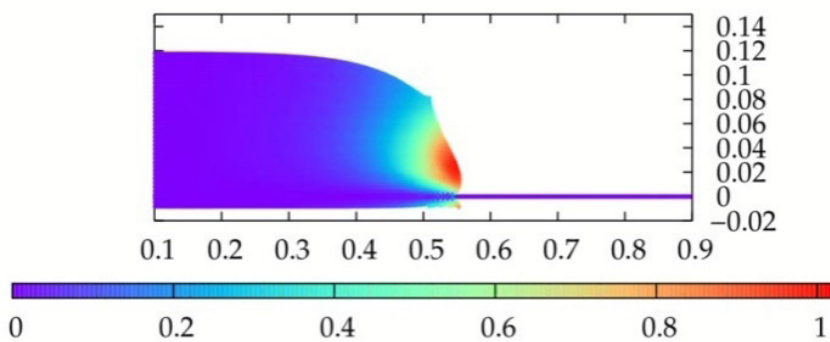

Figura 8 - Simulação numérica, em um determinado instante, com altura de ruptura: de $0,12 \mathrm{~m}$. A escala do gráfico indica a intensidade de velocidade $(\mathrm{em} \mathrm{m} / \mathrm{s})$

\section{CONCLUSÕES}

Apresentam-se, neste artigo, simulações de escoamentos de fluidos newtonianos, em regime laminar, utilizando um código SPH renormalizado, tanto em relação ao núcleo de suavização quanto ao seu gradiente.

Inicialmente, estudos de caso foram realizados e serviram como testes de validação do código. Entre estes testes, destacam-se o Poiseuille plano, o Poiseuille com superfície livre e o escoamento de Couette. Foram avaliados tanto aspectos transientes quanto permanentes, através da comparação teórico numérica da evolução do perfil de velocidade. Tal análise é possível mediante a utilização de fronteiras periódicas, que contribuem para a redução do domínio computacional, aliada com as condições de contorno de parede, aplicando a técnica das partículas fantasma.

Avaliam-se, também, duas formas distintas para representação da condição de contorno de aderência, considerando as 
velocidades das partículas fantasma nulas ou contrárias às suas contrapartes reais (Figura 3a). Para os estudos de caso realizados, o código numérico apresenta erros relativos máximos inferiores a $2 \%$ no perfil de velocidades quando comparados com a solução teórica, como pode ser visto nas Figuras 1, 3, 4 e 5.

Em relação ao problema tipo ruptura de barragem de fluido newtoniano, a evolução da frente da ruptura é reproduzida pelo código SPH corrigido, acompanhando a mesma tendência dos resultados experimentais (LEITE, 2009).

Observa-se que, para a resolução adotada, a resposta do código SPH renormalizado (corrigido) é adequada e concorda com as observações teóricas e experimentais, mesmo nas situações de partículas desordenadas e presença de superfície livre, como é o caso da ruptura de barragens. Em suma, o código desenvolvido e assim validado, é capaz de reproduzir problemas de Engenharia envolvendo fluidos newtonianos.

\section{AGRADECIMENTOS}

O primeiro autor agradece à FAPESP (Fundação de Amparo à Pesquisa no Estado de São Paulo) pelo financiamento de bolsa de doutorado (proc. 2009/00083-8) e ao projeto de parceria CAPES/FCT (Coordenação de Aperfeiçoamento de Pessoal de Nível Superior - Fundação para Ciência e Tecnologia - proc. BEX 5158/10-9) pela concessão de bolsa para estágio de doutoramento no exterior.

Os autores agradecem aos revisores pelas valiosas sugestões.

\section{REFERÊNCIAS}

BONET, J; LOK, T. S. L. Variational and momentum preservation aspects of Smooth Particle Hydrodynamic formulations.Computer Methods in Applied Mechanics and Engineering, v.180, n. 1-2, p. $97-$ 115, 1999.

CAPONE, T. SPH numerical modelling of impulse water waves generated by landslides. 2009. Tese (Doutorado) - Università di Roma La Sapienza, Roma, Itália, 2009.

COLAGROSSI, A. Ameshlesslagrangian method for free-surface and interface flows with fragmentation. 2004. Tese (Doutorado) Universitàdi Roma La Sapienza, Roma, Itália, 2004.

CUEILLE, P. V. Modélisation par Smoothed Particle Hydrodynamics des phénomènes de diffusion présentsdansunécoulement. 2005. Tese (Doutorado) - Institut National des Sciences Appliquées (INSA), Toulouse, França, 2005.

DILTS, G. A. Moving-Least-Squares-Particle Hydrodynamics I:consistency and stability. International Journal for Numerical Methods in Engineering, v. 44, n. 8, p. 1115-1155, 1999.

HOSSEINI, S. M.; MANZARI, M. T.; HANNANI, S. K. A fully explicit three-step SPH algorithm for simulation of non-
Newtonian fluid flow. International Journal of Numerical Methods for Heat \& Fluid Flow, v. 17, n. 7, p. 715-735, 2007.

LACHAMP, P. Modélisationnumérique de l'effet d'unobstaclesurlesécoulements de fluides à seuil par laméthode SPH. 2003. Tese (Doutorado) Université Joseph Fourier, Grenoble, França, 2003.

LAIGLE, D.; LACHAMP, P.; NAAIM, M. SPH-based numerical investigation of mudflow and other complex fluid flow interactions with structures. Computational Geosciences, v. 11, n. 4, p. 297-306, 2007.

LEITE, L. O. B. Determinação física e numérica de corridas de lama resultantes deruptura de barreira retendo material viscoplástico. 2009. Dissertação (Mestrado) - Faculdade de Engenharia de Ilha Solteira - FEIS/Unesp, Ilha Solteira, 2009.

MACIÁ, F.; ANTUONO, M.; GONZÁlEZ, L. M.; COLAGROSSI, A. Theoretical analysis of the no-slip boundary condition enforcement in SPH methods. Progress of Theoretical Physics, v. 125, n. 6, p. 1091-1121, 2011.

MONAGHAN, J. J. Simulating free surface flows with SPH. Journal of Computational Physics, v. 110, p. 399-406, 1994.

MONAGHAN, J. J. An introduction to SPH. Computer Physics Communications, v. 48, p. 89-96, 1988.

MONAGHAN, J. J. SPH and Riemann Solvers. Journal of Computational Physics, v. 136, p. 298-307, 1997.

MONAGHAN, J. J. Smoothed Particle Hydrodynamics. Reports on Progress in Physics, v. 68, n. 8, p. 1703-1759, 2005.

MONAGHAN, J. J. Smoothed Particle Hydrodynamic simulations of shear flow. Monthly Notices of the Royal Astronomical Society, v. 365, p. 199-213, 2006.

MORRIS, J. P.; FOX, J.; ZHU, Y. Modeling low Reynolds number for incompressible flow using SPH. Journal of Computational Physics, v. 136, p. 214-226, 1997.

OGER, G.; DORING, M.; ALESSANDRINI, B.; FERRANT, P. An improved SPH method: towards higher order convergence. Journal of Computational Physics, v. 225, p. 1472-1492, 2007.

RITTER, A. Die Fortpflanzung der Wasserwellen. Vereine Deutscher Ingenieure Zeitswchrift, v. 36, n. 2, p. 947-954, 1892.

RODRIGUEZ-PAZ, M. X.; BONET, J. A corrected Smooth Particle Hydrodynamics method for the simulation of debris flows. Numerical Methods for Partial Differential Equations, v. 20, n. 1, p. 140-163, 2004.

SHEPARD, D. A two-dimensional interpolation function for irregularly-spaced data. In: Proceedings of the 23rd ACM national conference, $A C M$ 68, 1968, p. 517-524. 
SOUTO-IGLESIAS, A.; GONZÁLEZ, L. M.; COLAGROSSI, A.; ANTUONO, M. SPH no-slip BC implementation analysis at the continuous level, In: 5th ERCOFTAC SPHERIC workshop on SPH applications, 2010.

TAKEDA, H., MIYAMA, S.; SEKIYA, M. Numerical Simulation of Viscous Flow by Smoothed Particle Hydrodynamics. Progress of Theoretical Physics, v. 92, n. 5, p. 939-960, 1994.

VASCO, J. R. G., MACIEL, G. F.; MINUSSI, C. R. Uma introdução às técnicas lagrangeanas: Uma aplicação do método SPH a problemas de engenharia. Revista Brasileira de Recursos Hidricos, v. 16, n. 1, p. 67-82, 2011.

VILA, J. P. Méthodes particulaires régularisées - développements récents etnouvelles applications. In: Proceedings ES AIM - 29ème Congres D'Analyse Numérique, 1998, p. 131-146. 